\title{
UNA MIRADA A LARGO PLAZO: ANÁLISIS MULTIDIMENSIONAL DE LA ACTIVIDAD PETROLERA EN ECUADOR
}

\author{
LOOKING AT THE LONG RUN: A MULTIDIMENSIONAL \\ ANALYSIS OF THE OIL ACTIVITY IN ECUADOR
}

\author{
Javier Martínez ${ }^{1}$ \\ Instituto de Altos Estudios Nacionales, Quito, Ecuador \\ Rony Parra $^{2}$ \\ Instituto de Altos Estudios Nacionales, Quito, Ecuador \\ Marcos Reis ${ }^{3}$ \\ Instituto de Altos Estudios Nacionales, Quito, Ecuador
}

Resumen: El artículo ofrece un análisis multidimensional de la explotación petrolera en Ecuador. Se discute la gobernanza petrolera en Ecuador y los impactos de la actividad en tres dimensiones: político-organizativa, económica y sociambiental. En resumen, se concluye que: i) los cambios en la gobernanza petrolera realizada en la última década fueron oportunos, pero la actividad no aparece como un vector de desarrollo a mediano y largo plazo para el país, ii) los conflictos sociambientales disminuyeron pero no se descarta que vuelvan a aparecer con virulencia si no se adopta una política clara respecto a esta actividad.

Palabras-clave: Gobernanza petrolera; economía petrolera; Impactos sociambientales.

\begin{abstract}
The article offers a multidimensional analysis of oil exploitation in Ecuador. It discusses the petroleum industry organization in Ecuador and its impacts in three dimensions: economics, social and environment. In summary, the key findings are: i) the regulatory changes in the oil sector in the last decade were good, but the activity cannot be considered as a development alternative for the country in the medium and long term, ii) the socio-environmental conflicts, especially in the Amazon area, decreased but not stopped in that period.
\end{abstract}

Keywords: Oil governance; Oil economy; Social and Environmental Impact.

1 Investigador del Instituto de Altos Estudios Nacionales (IAEN), Quito, Ecuador. Email: $<$ javier.martinez@iaen.edu.ec>.

2 Investigador del Instituto de Altos Estudios Nacionales (IAEN), Quito, Ecuador.

${ }^{3}$ Investigador del Instituto de Altos Estudios Nacionales (IAEN), Quito, Ecuador. Recibido en: 09.01.2016; aceito en: 01.05.2016. 


\section{INTRODUCCIÓN}

La explotación petrolera, así como otros recursos naturales estratégicos, asume un papel protagónico en la economía de muchos países del mundo en general y de América del Sur en particular, como, por ejemplo, Ecuador. El petróleo tiene una alta participación en la economía del país, generando impactos económicos, sociales y ambientales. Después del periodo de desregulación neoliberal con auge en los noventa, en especial después del inicio del gobierno de Rafael Correa en 2007, hay un cambio en la gobernanza del sector petrolero en Ecuador. La participación del Estado se expande y avanza desde la producción hasta la regulación. En ese sentido, ¿cuáles son los impactos económicos esperados de tal cambio? ¿Y los impactos socioambientales? ¿La actividad petrolera puede formar parte de una estrategia de desarrollo a largo plazo en Ecuador? Esas son algunas de las preguntas que este trabajo busca discutir.

Así, el objetivo de este artículo es contribuir a la reflexión en torno a los aspectos anteriormente citados desde un análisis multidimensional que englobe aspectos políticos, económicos y sociambientales. Consideramos esta propuesta muy pertinente porque en análisis específicos se pierde la perspectiva holística del problema, resultando en conclusiones parciales. La metodología utilizada para tal fin es el análisis crítico de la literatura y el uso de datos para un enfoque complementario cuantitativo.

Las principales conclusiones del trabajo pueden ser resumidas de tal modo: i) la actividad petrolera ha sufrido a lo largo de su historia de profundas carencias en su regulación y gobernanza; ii) a pesar de los cambios realizados en la última década encaminados a suplir estas carencias, un análisis económico demuestra las debilidades de esta actividad para que pueda suponer un vector de desarrollo a mediano y largo plazo; iii) los conflictos sociambientales han logrado ser reconducidos por el posicionamiento del Estado como actor de desarrollo en la región amazónica en la última década, pero si no se supera la actual posición ambigua ante esta actividad los conflictos volverán a posicionarse nuevamente; iv) en vista de estas conclusiones se apunta que no es suficiente con modificaciones en la gobernanza petrolera, si estas no están acompañados de profundos cambios de la economía del país.

El artículo está dividido en cinco secciones. En la segunda y la tercera secciones si discute la organización de la actividad petrolera en Ecuador, en especial los cambios regulatorios por los cuales el sector ha transitado en los últimos años durante el gobierno de Rafael Correa. La cuarta sección presenta un análisis de los datos referentes a la explotación petrolera en Ecuador, con enfoque en sus impactos en la economía y la sustentabilidad a largo plazo de la actividad. El quinto apartado avanza en el análisis multidimensional de la actividad petrolera, discutiendo sus impactos soioambientales en la región amazónica. Finalmente, la última sección, a modo de conclusión, realiza una serie de reflexiones y se propone unos interrogantes en torno al presente y futuro de esta actividad económica en Ecuador. 


\section{UNA BREVE HISTORIA DEL PETRÓLEO EN ECUADOR}

La historia de la explotación del Petróleo en Ecuador comienza a inicios del siglo XX. El primer pozo petrolero fue perforado en la región de la Costa en 1911. En los años siguientes la región de la Costa del país recibió muchas empresas transnacionales, como, por ejemplo, Texaco, Chevron y Shell.

Según la periodización de Parra (2015), la política petrolera en Ecuador atravesó varios cambios que se identifican en cuatro grandes periodos. Un primer momento, que inició a principios del siglo XX hasta el año 1971, con una subordinación de la gestión del sector petrolero a favor de las compañías trasnacionales y una ausencia de institucionalidad y de políticas públicas.

En 1967 Texaco perforó el primer pozo comercial en la Amazonía y desde entonces se tornó un actor principal en la explotación de petróleo en el país. Hasta el 1990, Texaco extrajo el $88 \%$ del total de la producción nacional de petróleo y operó el oleoducto. Además, perforó 399 pozos y construyó 22 estaciones de perforación (PETROECUADOR, 2011).

El segundo periodo coincide con los dos booms del petróleo que hubo en la década de 70 , entre 1972 y 1981. En este periodo se vivió un gobierno militar que marcó una clara tendencia nacionalista con políticas públicas direccionadas a la regulación y el control de la acción petrolera nacional. Los cambios establecidos a inicios de este período radicaron en la participación del Ecuador en los espacios energéticos internacionales y principalmente en la nacionalización de la empresa petrolera Gulf Oil que transfirió el monopolio del mercado interno a la Corporación Estatal Petrolera del Ecuador (CEPE) para que finalmente el Estado tome el control del consorcio CEPE-Texaco (FONTAINE, 2012).

El tercer periodo transita desde 1982 hasta el 2006 aproximadamente, y su rasgo característico es la apertura comercial que reformó el modelo económico. En este período se impulsa la privatización de los polos productivos que se encontraban bajo el protagonismo estatal, entre los que destacaba el sector petrolero. Así, la nueva política vinculada al neoliberalismo, y cristalizada en las recetas propuestas por el Consenso de Washington, enfatizó en el protagonismo de la inversión extranjera bajo el argumento de modernización del Estado (LLANES, 2007, p. 30).

Todavía dentro de este tercer periodo nace la empresa que asumirá un rol crucial en la explotación del petróleo en esta última fase. En septiembre de 1989 se crea PETROECUADOR conformando un holding, es decir, una matriz y seis filiales: tres permanentes (Petroproduccion, Petroindustrial y Petrocomercial), y tres temporales (Petropeninsula, Petroamazonas y Petrotransporte).

Finalmente, durante el gobierno de Alfredo Palacio en el 2006, y profundizado a partir del inicio del gobierno de la "Revolución Ciudadana" de Rafael Correa en 2007, se marca una nueva tendencia de la política petrolera en el Ecuador. Esta se caracteriza por varios cambios sustanciales en el sector. Con la intención de una mayor captura de renta petrolera se implantaron reformas normativas y se promulgó el Decreto Ejecutivo 1672, que expedía el Reglamento de la Ley 42 - 2006 reformatoria a la Ley de Hidrocarburos, cuyo fin fue reconocer al Estado con el 
$50 \%$ de los ingresos extraordinarios percibidos por el alza del precio del petróleo a partir del precio de referencia establecido en los contratos de explotación petrolera. Luego se terminó por reconocer el $99 \%$ de estos excedentes a favor del Estado (FONTAINE, 2012, p. 05).

Las trasformaciones establecidas en el sector petrolero se vieron acompañadas de un cambio normativo e institucional cuyo fin no solo fue reestablecer la influencia estatal en la política, regulación y control; sino también conseguir una participación directa a través del protagonismo de sus empresas públicas en el sector. Estos cambios, que serán analizados con más profundidad en el siguiente apartado, se vieron fortalecidos por la caducidad y salida de Occidental Petroleum Corporation (OXY) del país.

\section{LOS CAMBIOS EN LA GOBERNANZA DEL SECTOR PETROLERO EN LA ÚLTIMA DÉCADA}

En esa sub-sección vamos analizar los principales cambios a nivel de gobernanza que ocurrieron en el último de los cuatro periodos. En este se involucra el sector petrolero, desde el deseno y objetivos de los ministerios hasta el mayor protagonismo de las empresas públicas en las ejecuciones de políticas.

Uno de los principios que caracteriza a la política actual en el sector petrolero ha sido principalmente su nueva modelación en la estructura institucional, partiendo desde la división de las competencias del anterior Ministerio de Energía y Minas, el cual concentraba atribuciones tanto de electricidad, minería y de petróleos, a un ministerio solo dedicado al manejo del sector extractivo, el cual se denominó Ministerio de Recursos Naturales no Renovable (MRNNR).

Esta entidad hoy en día es la encargada, por un lado, del manejo del sector minero y por el otro del direccionamiento del sector de los hidrocarburos. Así, este nuevo ministerio se dividió en dos vice-ministerios: uno encargado del ámbito minero y el segundo del sector hidrocarburífero, dimensionando el aprovechamiento de los recursos naturales no renovables en su conjunto. En el sector petrolero se incorporó la visión también a la explotación de gas natural como parte de los hidrocarburos, creando una Subsecretaría de Gas Natural, con rango de vice ministerio, para su aprovechamiento.

Con la reforma a la Ley de Hidrocarburos, en julio de 2010, se terminó de estructurar el MRNNR y se fijaron las responsabilidades y competencias en la nueva institucionalidad propuesta con la creación de dos instituciones adscritas al ministerio. La primera es la Agencia de Regulación y Control Hidrocarburíferos $(\mathrm{ARCH})$ encargada de la regulación, fiscalización y control de la operación de las empresas públicas y privadas que actúan en el Upstream (exploración y explotación) y Downstream (transporte, refinación y comercialización) hidrocarbuirífero. La segunda es la Secretaria de Hidrocarburos del Ecuador (SH), encargada de la administración de las reservas y del manejo y suscripción de los contratos petroleros. Esta nueva lógica de ver el sector modificó las competencias de PETROECUADOR, que antes de la reforma se consideraba como la única empresa del Estado en el sector con el monopolio en el manejo y administración de contratos. Las transformaciones trajeron consigo una redistribución de responsabilidades, cuya configuración quedó como se presenta a seguir. 
En primer lugar, al MRNNR, con su brazo en el Viceministerio de Hidrocarburos, se circunscribieron a la rectoría en política hidrocarburífera y adjudicación de contratos en toda la cadena de valor de los hidrocarburos: i) exploración, ii) explotación, iii) transporte y almacenamiento, iv) refinación e industrialización y comercialización conjuntamente con los derivados y v) la terminación jurídica de los contratos en dichas fases.

Ya la SH administra en representación del Estado el potencial y las áreas hidrocarburíferas, suscribe los contratos, certifica las reservas y establece la participación de la renta en los contratos hidrocarburíferos. Además, es responsable del manejo de la información, así como de los bienes que sean revertidos al Estado por alguna eventualidad o infracción de la normativa.

Por fin, la $\mathrm{ARCH}$ regula y norma el sector, controla y fiscaliza las actividades y operaciones, e impone las sanciones a las infracciones hidrocarburíferas. Su gestión la cumple de manera desconcentrada para lo cual se adopta el concepto de Jurisdicción Hidrocarburifera, que se fundamenta en la existencia de los hidrocarburos, la infraestructura instalada para su aprovechamiento, y la comercialización de los combustibles y gas licuado de petróleo.

Estos cambios se identifican como instrumentos organizativos protagónicos, que son creados con el fin de ejercer la acción política del sector petrolero nacional para lo cual desempeñan funciones determinantes que ordenan los diferentes niveles de la política sectorial. Por un lado se observa un modelador en la regulación y control de la operación, y por el otro un eje de administración de los instrumentos contractuales en la cadena de valor hidrocarburífera, con lo cual ejercen un protagonismo en la aplicación de la política sectorial dictada por el MRNNR. La participación del Estado, entonces, va desde la planificación y regulación hasta la ejecución, que queda a cargo de las empresas públicas, que salieron muy fortalecidas en el periodo de la Revolución Ciudadana y en tal sector no es distinto. Por ejemplo, hasta marzo de 2013 se registraron un total de 26 empresas públicas de la Función Ejecutiva, y puntualmente en el sector petrolero se tiene a EP Petroecuador, Petroamazonas EP y las empresas mixtas Río Napo CEM y Refinería del Pacífico CEM.

Para entendernos el nuevo marco institucional del petróleo es necesario que se conozca cómo fue el rediseño del Estado en su conjunto. La modalidad de gobierno jerárquico del actual régimen se ve reflejado en el sistema institucional piramidal, en cuya cúspide se ubica la Presidencia de la República, seguido de dos organismos. El primero es la Secretaría Nacional de la Administración Pública (SNAP) la cual ejerce funciones de administración y de mejora de los procesos en la gestión del sector público. Y un segundo es la Secretaría Nacional de Planificación y Desarrollo (SENPLADES), en la cual se decanta la responsabilidad de planificación nacional a corto, mediano y largo plazo. Ésta, a través del "Plan Nacional del Buen Vivir" actúa como instrumento programático marcando el rumbo y dirección de las políticas públicas. Se trata de un actor al que se le ha dado un poder vinculante, en donde se concentra desde la priorización de las inversiones públicas hasta su participación directa en las decisiones estratégicas de las empresas públicas, al actuar por Ley en los Directorios de todas la empresa públicas de la Función Ejecutiva (LOEP, 2010). Entre ellas, las que pertenecen al sector petrolero.

Siguiendo la pirámide hacia abajo se encuentran siete ministerios de coordinación que son los encargados de crear y establecer una planificación intersectorial al mediano plazo. Estas 
carteras se encuentran repartidas en temáticas específicas con el fin de dar frente a los problemas levantados y observados por el Estado: i) sectores estratégicos, ii) política económica, iii) desarrollo social, iv) producción, v) seguridad interna y externa, vi) política, y vii) patrimonio natural y cultural.

Para el análisis de caso nos centraremos en la construcción de la agenda intersectorial del Ministerio Coordinador de Sectores Estratégicos (MICSE), el cual agrupa dentro de su seno y direccionamiento al Ministerio de Electricidad y Energías Renovables (MEER), MRNNR y el Ministerio de Telecomunicaciones (MINTEL). En el año 2013 además de estos ministerios también se unió el Ministerio del Ambiente (MAE) (PARRA, 2015). Esta institución actúa como un instrumento organizativo de política que reúne a variados actores y que facilita un marco de cooperación y de acción conjunta, presentados abajo en la gráfica 01.

De tal modo, la política sectorial viene anclada a la necesidad del Estado de la recuperación de lo público en el manejo sobre los hidrocarburos, llevando a una mayor captura de recursos a través de su gestión y explotación. No obstante, ninguna de estas instituciones son ejecutoras de estos principios, por lo que en este modelo esta facultad la ejercen las empresas públicas y privadas, actuando como instrumentos de actuación de las políticas públicas planteadas desde el poder Ejecutivo nacional (PARRA, 2015).

Así, uno de los puntos críticos dentro del modelo de gestión público petrolero ha sido la consolidación de una empresa estatal fuerte que pueda competir con el sector privado y que sea un actor que genere maximización de los recursos a partir su gestión eficiente. Como resultado de la aplicación de los distintos momentos de política practicados en el país, desde el fallido proceso de nacionalización en los setenta y una parcial aplicación del sistema privatizador en los noventa, se ha ocasionado en las empresas del Estado una configuración errónea de gestión, de tal forma que en ocasiones fueron quienes creaban la política, regulaban y controlaban su propia gestión.

Producto de esta fusión de modelos de administración en las empresas del Estado, en noviembre de 2007 la gestión de Petroecuador entró en una crisis, la cual se vio acrecentada por una relación problemática con las comunidades de la Amazonía que ocasionó el levantamiento y la protesta de varias de la provincia de Orellana que reclamaban atención en la construcción de obras e infraestructura comprometida por la empresa. Estas protestas paralizaron diversos campos petroleros, provocando una caída abrupta de producción de 175.000 barriles por día promedio de enero a noviembre de 2007 a 145.000 barriles por día al finalizar noviembre (NARVÁEZ, 2007).

En respuesta a la crisis de gestión de la empresa el Gobierno declaró su emergencia y terminó removiendo a su titular para pasar el mando de las operaciones de Petroecuador a la Fuerza Naval Nacional. Esta decisión fue el primer intento por restaurar el orden y mejorar la gestión de la empresa. Una vez al mando de la Fuerza Naval se contrató en marzo de 2008 los servicios de Wood Mackenzie, una consultora internacional que realizó la reestructura y promoción de un modelo empresarial. Este trabajo consistió principalmente en generar un modelo de gestión por procesos en cuya estructura las tres empresas filiales (Petroproducción, Petroindustrial y Petrocomercial) pasaron a ser unidades de negocio conformado por un staff de diversas gerencias. Fueron creadas siete gerencias: 1) exploración y producción, 2) transporte y almacenamiento, 3) refinación, 4) comercialización, 5) seguridad, 6) salud y ambiente, y 7) 
desarrollo organizacional. En la nueva estructura orgánica también se concibió como empresas subsidiarias de Petroecuador a Petroamazonas y Río Napo CEM (WOOD MACKENZIE, 2010).

Este proceso duró poco tiempo, pues mientras se implementaba el modelo sugerido en la estatal, varios acontecimientos iban marcando el rumbo final del modelo empresarial de Petroecuador. En 2009 se expidió la Ley Orgánica de Empresas Públicas (LOEP), y se creó en el sector petrolero una nueva configuración con dos empresas públicas. En el 6 de abril de 2010, mediante los Decretos Ejecutivos 314, 315, Petroecuador pasó a ser empresa pública para gestionar y actuar en todas las fases hidrocarburíferas, mientas que Petroamazonas se deslindó de Petroecuador y pasó a ser empresa pública dedicada exclusivamente a la exploración y explotación de hidrocarburos (Decretos Ejecutivo 314-315, 2010). O sea, el proceso de cambio propuesto por la consultora Wood Mackenzie fue interrumpido en la mitad.

Finalmente, con la salida de las Fuerzas Armadas de Petroecuador a inicios de 2010, y el cambio de ministro en el MRNNR en el 21 de abril de 2010, hay un nuevo cambio en la estrategia para el sector petrolero. Por la segunda vez en un periodo de dos años, se contrata una consultora internacional para que analice y direccione la gestión estatal en el mercado de los hidrocarburos. Bajo la dirección del nuevo ministro, el MRNNR contactó en Marzo de 2011 a la consultora Deloitte \& Touche, con el fin de volver a reestructurar las empresas públicas existentes y con miras a internacionalizarlas (EL UNIVERSO, 2011).

Como resultado del análisis realizado por tal consultora, se impulsó la creación de dos empresas independientes: la una dedicada al negocio del Upstream y la otra netamente al Downstream. Es así que Petroamazonas absorbió las áreas de exploración y producción de petróleo y gas natural, de acuerdo a su conocimiento del know-how, convirtiéndose en la única empresa pública en el país dedicada al Upstream hidrocarburífero. Petroecuador, por su parte, se quedó encargada de la operación aguas abajo, como es el mercado del transporte, refinación y comercialización nacional e internacional de los hidrocarburos (Decreto Ejecutivo N. 1351-A, 2013). De acuerdo a la posición del gobierno, este cambio propone buenas expectativas con respecto a mejorar los márgenes de eficiencia y eficacia de las empresas públicas, considerando la importancia de su gestión para los intereses del Estado (MICSE, 2013).

Es difícil evaluar empíricamente los resultados de la reestructuración de las empresas y sus impactos en la gestión, puesto que formalmente su aplicación se llevó a cabo hace poco tiempo, siendo la configuración actual inicialmente implementada en Enero de 2013. Todavía, analizando el proceso de reestructuración de las empresas públicas del sector hidrocarburífero, es eminente el sentido de premura del Ejecutivo por recomponer el sistema empresarial público del sector y hacerlo de alguna forma eficiente en su gestión.

Un análisis más detallado de los cambios en la regulación del sector petrolero será presentado en las conclusiones, cuando será posible entender tales transformaciones dentro de una óptica multidimensional donde se involucra aspectos económicos, sociales y ambientales resultantes de la actividad. Todavía, se puede destacar por ahora un resultado de los cambios hechos que es bien visible: un fuerte incremento de la producción petrolera de las empresas públicas concomitantemente a la bajada de la producción de las compañías privadas después del 2006 (gráfica 02). 
Una vez identificados los principales cambios en la gobernanza del sector petrolero en Ecuador - con énfasis en la última década - la siguiente sección se presenta algunos datos de la explotación petroleara en Ecuador que servirán de apoyo para la discusión sobre los posibles impactos, potencialidad y limitaciones de estos cambios del sector petrolero.

\section{LA EXPLOTACIÓN PETROLERA EN ECUADOR EN NÚMEROS}

En las últimas décadas, Ecuador ha incrementado su producción de petróleo, triplicando la producción anual en el periodo 1980-2014. En la última década, 2004-2014, la explotación se ha mantenido más o menos constante alrededor de 520 mil barriles por día, con un punto máximo en 2014 con 557 mil barriles (gráfica 03).

Como indica la tabla 01, Ecuador se ubica como el $28^{\circ}$ productor de petróleo a nivel global en 2014. De tal modo, entre los miembros de la Organización de Países Exportadores de Petróleo $(\mathrm{OPEP})^{4}$ Ecuador es el que menos produce, 557 mil barriles por día. También es posible analizar la Producción de petróleo per cápita y año. Tal indicador permite que se controle la producción de petróleo por el tamaño de la población. Eso es importante porque hay países que son grandes productores de petróleo pero cuentan con grandes poblaciones, así como países que no producen mucho pero tienen poca población. Con ese indicador Ecuador sale de la posición 28 para la de número 18 (indicado en la última columna de la tabla 01). Así, aunque Ecuador escale 10 posiciones en este indicador, aún está muy lejos de una situación como la de Qatar, Kuwait y Emiratos Árabes Unidos, por ejemplo. Tales países poseen una producción de petróleo muy alta con muy poca población.

Tal y como refleja la tabla anterior, Ecuador no presenta una producción de petróleo por persona lo suficientemente alta para poder contar con los ingresos de petróleo necesarios para mantener una renta per cápita alta de sus habitantes vinculada exclusivamente a dicha actividad (renta que es de carácter temporal hasta el agotamiento del recurso). Además, el horizonte temporal para el país con las reservas probadas de petróleo no es muy largo. Ecuador tiene $0.7 \%$ solo de las reservas de la OPEP, con 8,27 mil millones de barriles mientras Venezuela y Arabia Saudita poseen 299 y 266 mil millones de barriles en reservas probadas respectivamente. De tal modo, manteniendo la tendencia de producción de los últimos años, el país cuenta con petróleo como máximo para unas tres o cuatro décadas (grafica 4).

Si Ecuador no puede contar con el petróleo en el largo plazo, tampoco se puede considerar que la economía ecuatoriana estará bien en el corto plazo si se apoya únicamente en el sector petrolero. La alta volatilidad de los precios de los recursos naturales, en comparación a las manufacturas, vuelve mucho más difícil la planificación de largo plazo de las inversiones y gasto público en una economía. Otro aspecto que vuelve a los recursos naturales únicos en su análisis es que los precios de los mismos son, al revés de productos industrializados, decididos no por los productores sino por un mercado mundial financiero. Las mayores bolsas de commodities del

4 La Organización de Países Exportadores de Petróleo (OPEP) es una organización intergubernamental, con sede en Viena. Fue creada en 1960 por los mayores exportadores de petróleo entre los países en desarrollo. Los miembros actuales están discriminados en la gráfica 04. 
mundo están en Chicago (EEUU) y Londres (Reino Unido). O sea, los países productores en general no tienen mucha influencia en la determinación de los precios de los productos originados y explotados en sus países, sino que es el mercado financiero internacional el que los determina.

Así, con la actividad petrolera Ecuador queda dependiente de ingresos que son muy volátiles y con precios que no son determinados por los productores. El boom de commodities que se experimentó en el periodo 2003-2013 fue seguido por una fuerte bajada de los precios del petróleo en 2014 y, en especial, 2015. La gráfica 05 muestra la evolución de los precios de uno de los tipos de petróleo más operados en los mercados financieros, el West Texas Intermediate (WTI) en el periodo 2000-Octubre de 2015 (grafica 5).

Es importante destacar que Ecuador recibe, en general, menos que el precio del WTI. El crudo ecuatoriano sufre la aplicación del diferencial (castigo o descuento) por calidad respecto a su marcador internacional. Por ejemplo, según datos de la OPEP, en Septiembre de 2015, mientras el WTI presento un promedio de USD 45,48 por barril, dos de los principales tipos de petróleo que están presentes en Ecuador, el Crudo Oriente y el Crudo Napo, tenían como precio promedio USD 40,47 y USD 36,37 respectivamente.

Es necesario analizar más detenidamente los sectores en los cuales el país es competitivo en el escenario global, o sea, sus exportaciones. La participación del petróleo crudo en las exportaciones de Ecuador creció de manera considerable en los últimos 20 años, en especial debido al del boom del precio de commodities en el periodo 2003-2013. Por ejemplo, según datos de la Conferencia de las Naciones Unidas sobre Comercio y Desarrollo (CNUCYD o UNCTAD, en inglés), en 1995 el petróleo crudo representaba 27\% del total de las exportaciones del país. En 2002, justo antes del inicio de la fuerte subida en el precio de los recursos naturales, representaba 32\% del total. Desde 2008 hasta 2013 el petróleo crudo representó más de 50\% de las exportaciones del país, llegando al 51\% en 2013.

Se espera que la presencia del petróleo crudo en las exportaciones de Ecuador disminuya en los próximos años debido al efecto resultante de la bajada global del precio de los recursos naturales que empezó en 2014 y se volvió más acentuada en 2015. Asimismo, es importante destacar que una de las consecuencias del crecimiento de la participación del petróleo crudo en la pauta exportadora de Ecuador fue la disminución en la diversificación y complejidad de las exportaciones. Según el "Atlas de la Complejidad Económica", el país, que ya tenía una complejidad económica muy baja, cayó en el Ranking Global, pasando de la posición $95^{\circ}$ (de un total de 123 países) en 2003 para la posición $100^{\circ}$ en 2013.

El ranking considera los distintos grados de complejidad de las economías. Como todos los bienes requieren de conocimientos y capacidades específicas, cuanto más complejo son los productos que una economía pueda producir, más probable que estos tengan alto valor añadido y sean capaces de generar spillovers para otros sectores, posibilitando la creación de productos aún

\footnotetext{
5 The Atlas of Economic Complexity fue inicialmente publicado en 2011 (Hausmann et al, 2011) por el economista Ricardo Hausmann de la Universidad de Harvard y el físico César Hidalgo del Instituto Tecnológico de Massachusetts. A partir de entonces, el índice de complejidad económica elaborado en esta publicación, ha ganado relevancia para estudios de temas de desarrollo y capacidad de crecimiento económica de los países, en especial los países en desarrollo. Para más informaciones, véase http://atlas.cid.harvard.edu/
} 
más complejos en el futuro. De tal modo, en un ejercicio de proyección para el futuro del crecimiento de las economías basado en sus capacidades de producir y crear productos complejos, los autores indican que para el decenario 2014-2023, la Economía de Ecuador crecerá en promedio $2.1 \%$, ocupando la posición de numero 15 de 19 en América Latina y 98 en el ranking global (en un total de 124 naciones).

En el siguiente apartado se partirá de las diferentes fases ya descritas por la que ha transitado la gobernanza del sector petrolero y las dinámicas económicas descritas en este apartado para analizar la conflictividad socioambiental en la Amazonía ecuatoriana. Esto nos permitirá reflexionar en torno a los impactos ambientales y sociales de todos los procesos políticos y económicos analizados.

\section{GOBERNANZA PETROLERA Y CONFLICTOS SOCIOAMBIENTALES EN LA AMAZONÍA ECUATORIANA}

Como se ha explicado, la gobernanza petrolera en Ecuador ha pasado por cuatro grandes fases con límites temporales borrosos entre ellas. La primera abarca la explotación petrolera de la Península de Santa Elena, en la costa, que comenzó a principios del siglo XX y las exploraciones petroleras en la Amazonía. Ésta se caracteriza, como se ha explicado, por una ausencia total del Estado en la regulación de estas actividades. En 1967 Texaco perfora su primer pozo en la Amazonía. La actividad petrolera pasará de ser una actividad económica secundaria en el país a ser la principal entrada de divisas en poco tiempo. Debido a este protagonismo, que acarreó grandes transformaciones económicas y sociales, así como fuertes impactos socioambientales, este apartado se va a centrar en la explotación en la región amazónica.

Podemos decir, por tanto, y relacionándolo con la temporalidad anteriormente descrita, que la actividad petrolera ha transitado en el Oriente ecuatoriano por tres grandes etapas de gobernanza con consecuencias socioambientales diferentes. En la primera, a lo largo de los años setenta, con un gobierno militar nacionalista pero débil económicamente, el Estado intentó imponer una regularización de la actividad y nacionalizó las reservas. Pero su debilidad, reflejada en una fuerte dependencia económica y tecnológica del exterior, le llevó a tener que depositar todo el peso de la extracción en una empresa como Texaco. Se podría hablar así de la "era Texaco" (JUTEAU-MARTINEAU et al., 2014). El segundo gran periodo comienza con la liberalización de la economía por los ajustes estructurales de la década de los ochenta en adelante, lo que significó la entrada de un gran número de empresas y conllevó un aumento exponencial de los conflictos sociales, algunos de ellos heredados de la "era Texaco" pero que comienzan a explotar en estos momentos. La última etapa comienza con el colapso de la economía ecuatoriana en el 2000, se fortalece tras la salida forzada de la presidencia del país de Lucio Gutiérrez en abril de 2005 y la llegada de Alfredo Palacios a la misma, y finalmente se consolida con la llegada al poder en 2007 de Rafael Correa y su Revolución Ciudadana. Esta fase está caracterizada por una reorientación de las reivindicaciones de los actores sociales hacia políticas de desarrollo, un discurso de fuerte carácter nacionalista y un aumento de la inversión pública para políticas sociales. 
Es importante tener en cuenta que a partir de los cincuenta comenzaron a promoverse a nivel internacional una serie de políticas que englobamos bajo el nombre de "desarrollismo clásico", donde se consideraba al Estado como un agente modernizador imprescindible para lograr el cambio político, económico y social (Paz y Miño, 2007). A partir de 1960, con las Fuerzas Armadas a la cabeza, se comienza una serie de reformas estructurales encaminadas a insertar a Ecuador de manera definitiva la etapa desarrollista (BARRERA, 2001, p. 82).

Las políticas desarrollistas alcanzan su punto más álgido en Ecuador con el gobierno del general Guillermo Rodríguez Lara, que sube al poder en 1972. Este gobierno contó con grandes recursos, por el comienzo de la explotación del petróleo, y promovió un programa de medidas "nacionalista y revolucionario". Así, esta etapa representó el esfuerzo más articulado de desplegar un patrón de desarrollismo estatal en Ecuador y fomentar un modelo de Industrialización Sustitutiva de Importaciones (ISI). A su favor jugaban los recién explotados recursos petroleros amazónicos que transformaron la economía nacional ${ }^{6}$. Los cuatro grandes ejes de actuación de este gobierno fueron: el impulso al modelo ISI, la profundización de la reforma agraria expidiendo una nueva Ley de Reforma Agraria en 1973, la reformas tributaria y del Estado (Barrera, 2001: 83). Así, el inicio de la explotación petrolera permitió dar un salto en las exportaciones, pero no logró su diversificación, convirtiéndose, una vez más, en uno de los productos primarios de los que ha dependido la economía del país. Ecuador entró, a pesar de los ingresos petroleros, en una espiral de endeudamiento y, en un contexto de crédito fácil, se vio obligado a financiar por esta vía hasta los gastos corrientes. A lo largo de la década de los setenta la deuda externa se multiplicó por 22, pasando del 16\% al 42\% del PIB (ACOSTA, 2006, p 119124). En 1981, el peso de tener que hacer frente a la crisis de la deuda, potenciado por una caída de los precios del petróleo, era ya demasiado elevado. El fenómeno climático del Niño (que destruyó numerosas infraestructuras, entre ellas muchas petroleras, lo que significó una caída de la producción de crudo) y la muerte del presidente del país, Jaime Roldós, en un accidente en mayo, acrecentaron las dificultades del gobierno y llevaron en 1982 a tomar las primeras medidas de ajuste (Burbano, 2006: 302). A partir de entonces se produce una transición hacia el modelo neoliberal que se consolidará en la década de los noventa.

En la Amazonía, aunque distante de los centros de poder y siempre receptora tardía de sus dinámicas, esta etapa desarrollista marcó su evolución. Varios hitos acabaron por incorporar de manera definitiva esta región al Estado nacional. Por un lado las reformas agrarias fueron decisivas a la hora de promover una colonización efectiva y la ampliación de la frontera agraria en la alta Amazonía ecuatoriana, y por otro, el comienzo de la explotación petrolera, posibilitó la construcción de infraestructuras en los gobiernos desarrollistas. Así, la importancia que hemos visto que adquirió para el gobierno militar nacionalista la entrada de divisas petroleras y la dependencia de su proyecto nacionalista de las mismas, acabó instaurando un modelo de dominación del petróleo sobre el resto de los intereses del territorio. La nefasta convergencia de tres factores, la desinformación de las comunidades ante una nueva actividad, los intereses

\footnotetext{
${ }^{6}$ La exportación de petróleo de la Amazonía comenzó a fluir en agosto de 1972. Un año después el precio del barril del "crudo Oriente" pasó de 3.83 a 13.14 \$. Este hecho posibilitó que el gobierno de Guillermo Rodríguez Lara controlara un excedente económico que le permitió un aumento del gasto corriente del $12 \%$ anual y de inversiones públicas del $8,4 \%$, fomentando un enorme crecimiento económico del sector público que aumentó la participación del Estado en el Producto Interior Bruto (PIB) de un 9,5\% en 1965 a un 22.5\% en 1980 (Montúfar, 2000: 31).
} 
económicos del gobierno y la dependencia absoluta de una empresa privada (Texaco), potenció, como veremos, los efectos negativos de dicho modelo.

El modelo desarrollista conllevó a que tres dinámicas diferentes se potenciaran entre ellas en la Amazonía. La llegada de las petroleras generó infraestructuras y abrió carreteras que permitieron la colonización de tierras amazónicas para la ampliación de la frontera agrícola tan necesaria en la Reforma Agraria del 1973. Por otro lado, la colonización de nuevas tierras y la actividad de Texaco potenciaron la llegada de las madereras, pues por un lado Texaco colabora con ellas para abrir rutas y desbrozar áreas, y por otro el bosque primario es explotado en las tierras recientemente colonizadas alrededor de las rutas. Así nos encontramos con una de las principales consecuencias de esta primera etapa. De ser territorios ancestrales de diferentes grupos étnicos, parte de la región amazónica pasó a ser una zona de explotación intensiva de petróleo, de tierras agrícolas y de recursos madereros, una auténtica frontera económica. Todo esto se realizó, además, sin ninguna organización territorial ni urbanística en un momento que se produjeron grandes cambios demográficos en número y composición. Este crecimiento demográfico, que fue bastante amplio debido a la colonización favorecida por la apertura de tierras, fue aún mayor alrededor de las infraestructuras petroleras. Este aumento poblacional en las áreas de influencia de las explotaciones petroleras incrementó enormemente los problemas sanitarios por la falta de servicios básicos (debido al crecimiento urbanístico no planificado) y la exposición continua a un ambiente altamente contaminado (BUSTAMANTE Y JARRÍN, 2007).

La coincidencia de la gestión pública de la actividad petrolera por parte del gobierno nacionalista con el Ministerio del Petróleo y la Corporación Estatal del Petróleo Ecuatoriano (CEPE) (hoy Petroecuador EP), la necesidad de divisas del proyecto desarrollista y la dependencia de la empresa Texaco en la actividad de producción, conllevó una estrategia maximizadora de la renta petrolera reduciendo al mínimo los costos de producción. De esta manera tanto la estrategia productiva como la competencia de la gobernanza local estuvo en manos de la empresa, exponiendo a las poblaciones locales a altos grados de contaminación derivados de una estrategia que favorecía tecnologías obsoletas y bajo mantenimiento de las infraestructuras (JUTEAU-MARTINEAU et al., 2014). Gran parte de estos impactos sanitarios y ambientales salieron a la luz 30 años después en el macrojuicio a Texaco.

A partir de los años ochenta, el surgimiento con fuerza de las organizaciones indígenas, y su vinculación a movimientos internacionales de carácter ecologista, potenciaron una opinión pública cada vez más formada y crítica con la actividad petrolera. En 1993, 30.000 habitantes de las áreas de influencia de la actividad de Texaco presentaron una denuncia a la empresa por daños sanitarios y ambientales en un tribunal de la ciudad de New York, sede de Texaco. El 16 de agosto de 2002, la Corte de Apelaciones del Distrito Sur de New York, después de nueve años de batalla legal, resolvió que la encargada de enjuiciar este hecho era la justicia ecuatoriana y que la empresa debía de someterse y acatar a la misma. El 07 de mayo del 2003, los demandantes iniciaron las acciones legales contra la petrolera en la Corte Superior de Justicia de Sucumbíos. En el 2011 la Corte de Loja impone finalmente a la empresa Chevron (que adquirió Texaco en 2001) una multa de 9.500 millones de dólares por el derramamiento de más de 80.000 toneladas de residuos petrolíferos durante los años 1964 y 1992. Luego de 10 años de litigio ante las Cortes ecuatorianas, y después de pasar por dos instancias inferiores, el 12 de noviembre de 2012 la Corte Nacional ratifica la sentencia contra Chevron Corporation por todos los daños ambientales y dobla la suma impuesta en el 2011 por no pedir disculpas ni reconocer los hechos. A pesar de 
estas victorias ante los tribunales, la compañía se niega a reconocer la sentencia y en su lugar ha decidido acusar a sus víctimas de extorsión (PRIETO, 2014).

Con el proceso de liberalización de la economía ecuatoriana iniciado en los ochenta, vinculada al fracaso del modelo desarrollista, aterrizan numerosas empresas al sector de la producción petrolera. Esto implica el paso de una cierta paz social negociada a una relación conflictiva (JUTEAU-MARTINEAU et al., 2014). Por un lado la paz que acompañó a la era Texaco se rompe. En un principio, la estrategia de relaciones comunitarias de la empresa, el desconocimiento de la población de los efectos de la actividad y el apoyo del ejército minimizó los conflictos y permitió anticipar y contener las potenciales rebeliones. Pero después de la liberalización la licitación de bloques se multiplicó, ampliando la frontera petrolera y avanzando la misma hacia el centro-sur, donde las comunidades, ya informadas de lo ocurrido en los territorios donde operó la Texaco, se comenzaron a organizar ante su llegada. Es en este momento donde comienza el largo conflicto del bloque 23 licitado a la petrolera argentina CGC con la comunidad de Sarayacu (FONTAINE, 2012).

Este momento coincide además con la emergencia del actor étnico. Como hemos visto, el régimen desarrollista de los setenta pronto pierde terreno con el neoliberalismo a inicios de los ochenta. La idea de un Estado planificador, que se erigiera como el principal agente de desarrollo y ente regulador, chocaba de pleno con las políticas desregulacionistas que imponía el nuevo paradigma económico. La fuerte crisis económica y el proceso de desmantelamiento del Estado activaron la conflictividad entre los diferentes grupos de poder y actores sociales. Todo esto tuvo un fuerte impacto en la morfología del Estado, debilitándolo enormemente y, como consecuencia de ello, provocando una discontinuidad en sus políticas y una constante desinstitucionalización. El Estado ecuatoriano a principios de los noventa recordaba a un mosaico de diferentes "nichos" políticos vinculados a los diferentes grupos de poder (BARRERA, 2001). Así, la llegada del neoliberalismo a Ecuador traería consigo una sociedad empobrecida y fragmentada, con un sistema político debilitado y sin un proyecto nacional ni hegemonías claras. En este contexto el país entró en la década de los noventa conmocionado por el primer gran levantamiento indígena contemporáneo. A partir del 3 de junio de 1990 y por alrededor de nueve días, miles de indígenas ocuparon las carreteras de la Sierra y las principales ciudades hasta llegar a la capital, Quito, y tras ocho días de protestas obligaron al presidente Rodrigo Borja a sentarse a negociar los 16 puntos del Mandato por la vida de la Confederación de Nacionalidades Indígenas del Ecuador (CONAIE). Así es como irrumpió con fuerza en el panorama nacional la CONAIE, que había sido fundada en 1986. Esta irrupción y su posterior consolidación a lo largo de los noventa situaron al movimiento indígena como un potente e importante actor social en el panorama político ecuatoriano. La irrupción del movimiento indígena posicionaría la "cuestión étnica" como una de las principales prioridades de la agenda de las agencias de cooperación y de los poderes públicos. Este proceso se dio en un principio en la Sierra, aunque muy pronto organizaciones indígenas de otras regiones, como la Organización de Pueblos Indígenas de Pastaza (OPIP), adquirieron, como veremos, una fuerte notoriedad ${ }^{7}$. Así como en la Sierra la lucha principal había sido por la tierra, en la Amazonía su principal reivindicación y lucha sería

\footnotetext{
${ }^{7}$ Nos referimos al proceso de adquisición de notoriedad pública, no a su génesis. Más allá de la evolución de los diferentes movimientos indígenas del país, lo que está claro es que el levantamiento en la Sierra de 1990 puso al movimiento indígena en la arena política a nivel nacional y que más tarde ese tirón fue aprovechado por otros movimientos como la OPIP en 1992.
} 
por los territorios. Ante los importantes cambios que como se ha explicado sucedieron en esta región en las décadas de los setenta y ochenta, vinculados principalmente a la explotación petrolera, colonización y ampliación de la frontera agraria, los pueblos indígenas amazónicos comenzaron a organizarse. Con estos antecedentes, a partir de los setenta surgieron organizaciones de carácter indígena, especialmente en las provincias de Napo y Sucumbíos, las áreas más pobladas en esa época, como la Federación de Comunas Unión de Nativos de la Amazonía Ecuatoriana (FCUNAE), la Federación de Organizaciones Indígenas del Napo (FOIN) y la Federación de Organizaciones Indígenas de Sucumbíos del Ecuador (FOISE).

En esta nueva coyuntura, los conflictos aumentan exponencialmente y la resistencia se desarrolla tanto como medio de contestación antes los impactos ya observados de la actividad petrolera, como medio de negociación de las indemnizaciones con las empresas. Las organizaciones indígenas, apoyadas en toda una red de ONG de todo tipo tanto nacionales como internacionales, permiten desarrollar una gran presión sobre las empresas, en especial sobre Petroecuador que había asumido en 1992 los pozos de la Texaco. Durante esta etapa conflictiva nos encontramos con posiciones cada vez más polarizadas. Por un lado el Estado ecuatoriano con licitaciones cada vez más numerosas que amplían la frontera petrolera, y por otra los actores sociales, básicamente indígenas y ecologistas, que piden una moratoria de la actividad petrolera. Dos ejemplos claros de la conflictividad de este momento es la lucha en el bloque 10 de ArcoAgip y el ya mencionado conflicto del bloque 23 de Sarayacu.

Pero las organizaciones indígenas pronto comenzaron a enfrentar una debilidad vinculada tanto al cansancio organizativo como a sus divisiones internas (sobre todo con la entrada en la política formal en 1995 a través del partido Pachakutik), así como a las posiciones intransigentes de las organizaciones ecologistas que habían sido sus primeras aliadas. Esta debilidad tuvo como resultado que la conflictividad global de esta época no lograra imponer una reforma profunda de la gobernanza petrolera. A pesar de que todas estas luchas lograran la adopción de varias leyes de protección de los derechos humanos y el ambiente (la Ley de Gestión ambiental en 1999, el Reglamento Ambiental para las Operaciones Hidrocarburíferas de 2001 y el Texto Unificado de la Legislación Ambiental en 2003), no se logró influir en los ejes centrales de la toma de decisiones para posicionar las demandas de los pueblos amazónicos en la gobernanza petrolera (JUTEAU-MARTINEAU et al., 2014).

Entre el 2001 y el 2007 la situación conflictiva continúo y una serie de eventos y de conflictos supuso el principio de un giro en las posiciones de los diferentes actores ante la gobernanza petrolera. En 2001 se constituye la asamblea biprovincial de Orellana y Sucumbíos, que supone una radicalización de la protesta al coordinar a nivel biprovincial acciones como bloqueos, paros y ocupaciones de infraestructuras. Ante esta radicalización, la postura del Estado también se radicaliza con medidas cada vez más duras en la gestión de los conflictos. Esta situación termina con el dramático conflicto del 2007 en Dayuma de la que ya se habló anteriormente. A partir de esta fecha los conflictos comienzan a individualizarse y se dirigen cada vez a la búsqueda de compensaciones e indemnizaciones, siendo instrumentalizados por las comunidades para dar más valor económico a sus demandas.

En este contexto es en el que llega Rafael Correa y su Revolución Ciudadana al poder en el 2007, en lo que supone el retorno estatal a la escena pública. El nuevo proyecto requerirá de gran cantidad de recursos económicos, lo que se plasmará en una nueva ofensiva extractivista. 
Esta vuelta del Estado se fundamenta en el restablecimiento de las capacidades de planificación del desarrollo y regulación de la economía por parte del Estado, en lo que podría conceptualizarse como un proyecto neodesarrollista (RAMÍREZ Y MINTEGUIAGA, 2007). En él, el restablecimiento de la planificación estatal en la estrategia nacional de desarrollo juega un papel crucial, hecho que se materializa en la dotación de responsabilidades, recursos y toma de protagonismo de la Secretaría Nacional de Panificación y Desarrollo (SENPLADES). La llegada al poder de la Revolución Ciudadana inicia un periodo de transformaciones institucionales y políticas con la voluntad de aumentar la capacidad de influencia en las políticas sociales. Se pretende reconstruir el Estado después de su desmantelamiento en la etapa neoliberal, continuando o incluso aumentando para ello con la explotación petrolera y minera, pero intentando aportar respuestas a las demandas económicas, socioculturales y ambientales de la población. Así, la nueva política pasa por la conquista, después de su desaparición por décadas si es que alguna vez existió, de la legitimidad del Estado como actor de desarrollo en la región amazónica como mecanismo de lograr la paz social, en un contexto de explotación petrolera a la que no renuncia.

El programa político de la Revolución Ciudadana se apoyó en sus inicios en dos documentos fundacionales: la Constitución de Montecristi de 2008 y el Plan Nacional del Buen Vivir. En los dos se da un protagonismo a los derechos sociales y, por primera vez en la historia de la legislación internacional, a los derechos de la naturaleza. Imbuidos en la filosofía de estos documentos que caracterizaron los primeros años de la Revolución Ciudadana, y recogiendo las demandas históricas de la sociedad civil, en el 2007 el gobierno lanzó la iniciativa Yasuní ITT. El 4 de abril de 2007 el presidente Rafael Correa firmó un memorando de entendimiento con el presidente de Brasil, Luiz Inácio Lula da Silva, para la participación de Petrobras, empresa estatal brasileña, en el desarrollo del campo petrolero 43 en el Yasuní (conocido como ITT). Como respuesta a dicho memorando, un sector del Gobierno con fuerte influencia del ambientalismo presentó, en manos del entonces Ministro de Energía y Minas, intelectual de izquierdas ecuatoriano y próximo a los movimientos conservacionistas (ACOSTA, 1995), una contrapropuesta que consistía en reclamar una compensación económica internacional por no extraer el petróleo, que suponían el $20 \%$ de las reservas probadas del país. Esta compensación vendría a reparar, en parte, las pérdidas que dicha decisión generaría a las arcas del Estado necesitadas de estos recursos, puesto que esta decisión beneficiaría a toda la humanidad y no sólo a Ecuador. Finalmente, el Gobierno se decantó por esta segunda opción, conocida como Proyecto ITT Opción 1, y fue presentada en la Asamblea General de la ONU el 24 de septiembre de 2007 por el presidente Rafael Correa (ACOSTA Y MARTÍNEZ, 2010). Dicha propuesta consistía en el establecimiento de un mecanismo de compensación económica en forma de fideicomiso internacional, que resarciera en un 50\% lo que Ecuador dejaría de percibir por mantener sus mayores reservas petroleras permanentemente sin explotar. Este proyecto se topó desde el principio con numerosos problemas, generados básicamente por las contradicciones internas dentro del Gobierno entre el discurso ambientalista de una parte minoritaria del mismo y la apremiante necesidad de los recursos económicos provenientes del petróleo que el proyecto neodesarrollista necesitaba. La necesidad se fue imponiendo y el discurso ambientalista gubernamental fue diluyéndose, llevándose con él a sus defensores. Ya para 2010 había gente que daba por muerto el proyecto y que se preguntaba incluso si alguna vez había tenido vida real (AGUIRRE ANDRADE, 2010, p. 66-67). Finalmente, el presidente Correa dio por finiquitado el 
Proyecto ITT Opción 1 el 15 de agosto de 2013 en un discurso retransmitido por televisión, con la argumentación de que la comunidad internacional no había mostrado suficiente interés.

La puesta en marcha de esta iniciativa muestra la fuerte capacidad que se fue adquiriendo desde los ochenta de movilización de los actores sociales y las comunidades amazónicas, pero su fracaso demuestra también una falta de regulación de la explotación y el uso de la renta petrolera. La desregularización ya comentada de la economía ecuatoriana a partir de los ochenta llevó al desplome de la economía del país influenciada por la fuerte dependencia del recurso petrolero. A pesar de la cantidad de divisas que entraron por el petróleo, como se ha explicado anteriormente la economía no logró diversificarse, quedando en un grado de complejidad muy pequeño sin observarse un impulso en el desarrollo social y económico. Aunque el nuevo gobierno ha pretendido superar este reto intentando lograr un cambio en la matriz productiva, su éxito ha sido muy limitado.

El gobierno actual se encuentra, pues, ante varios desafíos importantes. Por un lado lograr disminuir la dependencia de la renta petrolera que, como se ha explicado, no asegura un buen futuro ni a corto, medio o largo plazo. Por otro lado tiene que afrontar el desafío socioambiental sin las ambigüedades actuales, lo que implica la reparación de los activos de los activos sociales y ambientales, intentando conjugar el desarrollo de la región amazónica con la preservación de las culturas locales. Finalmente, y para poder salir de la ambigüedad anteriormente mencionada, sería imprescindible establecer claramente los límites de aceptabilidad de la explotación petrolera.

\section{CONCLUSIONES}

El artículo hizo un análisis multidimensional de la explotación del Petróleo en Ecuador, con énfasis en el periodo más reciente. Se pudo verificar que desde el inicio del gobierno de la revolución ciudadana de Rafael Correa, en 2007, hay un fuerte cambio en la organización del sector petrolero. El Estado deja su papel de coadyuvante y asume un protagonismo hasta entonces nunca observado en el país. De hecho, se hizo la reestructuración de las instituciones en el sector petrolero y su configuración a partir de un ministerio dedicado exclusivamente a la política extractiva. Además, se crearon entidades adscritas a fiscalizar las actividades en la operación y para el manejo de las reservas. Eses hechos dan a notar la intención del Estado sobre la apropiación de la gestión del sector petrolero tanto en la directriz de sus políticas como en su regulación y control.

Eses cambios generaron aspectos positivos y negativos. Entre los positivos, se puede destacar el reconocimiento de que el petróleo es un recurso natural estratégico. De tal modo, el Estado debe explótalo dentro de un contexto de una política pública que contemple no solo la eficiencia, pero también la sustentabilidad, impactos ambientales y sociales y el destino de la renta generada. Así el gobierno puede coordinar, planificar y priorizar inversiones de proyectos a largo plazo en la integridad de la cadena de valor, desde la extracción hasta la comercialización, buscando hacer con que la economía rompa el path-dependence en relación a la venda de crudo y pueda caminar hacia un desarrollo económico y social sostenible. 
Todavía, hay aspectos que pueden ser considerados negativos también. El primer es que el modelo interventor del Estado podría relegar y entorpecer la acción privada en la operación del upstream, disminuyendo el poder de generar empleo y renta de la cadena petrolera. Para que eso no ocurra, es necesario que el gobierno ponga reglas claras y que incentiven al sector privado a actuar también, complementando la acción del sector público. Por ejemplo, el gran número de cambios realizados en la gestión de la Petroecuador, como discutido en la sección 2, son un ejemplo de cómo el gobierno puede generar incertidumbre, disminuyendo la posibilidad de inversiones de largo plazo en el sector. Por o tanto, hay que se fijar reglas claras y buscar su manutención; una política errática no es deseable y puede tener efectos contrarios al esperado.

Además, en la sección 3 se discutió algunos de las principales cifras asociadas a la actividad petrolera en el país y sus impactos en la estructura productiva, desarrollo económico y sustentabilidad del crecimiento económico. De los números presentados se pueden extraer las siguientes conclusiones: i) Ecuador ha logrado aumentar su producción petrolera en las últimas décadas, pero la falta de reservas hace que sea imposible contar con esta actividad a largo plazo en el país, ii) la volatilidad del precio de los recursos naturales y su aspecto geopolítico, con el precio asignado en los mercados financieros con sede en países avanzados, hacen que a corto y mediano plazo la explotación del petróleo no sea la puerta de salida para el desarrollo económico y social y iii) la falta de diversificación en las exportaciones se traduce en una economía con baja complejidad económica y, consecuentemente, poca capacidad de innovación, elemento crucial para la creación de una economía sostenible y con alto nivel de ingresos a largo plazo. De tal modo, del punto de vista económico, la explotación del petróleo en Ecuador no parece ofrecer soluciones ni para el corto ni mediano ni largo plazo.

Por fin, la sección 4 hemos visto como tras varias décadas de conflictos socioambientales relacionados con la actividad petrolera, Ecuador sigue sin resolver las tensiones vinculadas a la gobernanza petrolera. Por un lado sigue dependiendo de los ingresos petroleros, lo que ha orientado la política hacia la Amazonía de la política de la Revolución Ciudadana. Por otro lado se busca hacer frente a los problemas sociales y ambientales derivados de esta actividad en un contexto donde los actores sociales aparecen muy divididos y con estrategias cada vez más individualistas. El fracaso de la propuesta Yasuní ITT muestra, por un lado, la dependencia de Ecuador del petróleo, y por otro lo negativo que pueden resultar las tensiones entre diferentes políticas públicas en un contexto de ambigüedad política. Por un lado, el movimiento ecologista que en un principio tenía fuerza dentro de la Revolución Ciudadana pretendía hacer de la iniciativa Yasuní ITT un ejemplo de la lucha contra el cambio climático. Por el otro lado, el gobierno neodesarrollista no estaba dispuesto a asumir el costo económico de la medida en un contexto de gran dependencia de esta actividad y escasa complejidad económica. Al oponer una utopía ecológica con una utopía desarrollista, este debate no hace más que potenciar las deficiencias de la gobernanza petrolera en el país. Deficiencias que es necesario resolver si se quiere evitar otro ciclo de conflictividad socioambiental en el futuro. 


\section{BIBLIOGRAFÍA}

ACOSTA, Alberto. Breve historia económica del Ecuador. Quito: Corporación Editora Nacional, 1995.

ACOSTA, Alberto; ESPERANZA, Martínez (Eds). ITT-Tasuní: entre el petróleo y la vida. Quito: Abya Yala, 2010.

AGUIRRE ANDRADE, Milagros. La selva de papel. Quito: CICAME, 2010.

BARRERA, Augusto. Acción colectiva y crisis política. El movimiento indígena ecuatoriano en la década de los noventa. Quito: Abya-Yala y OSAL/CLACSO, 2001.

BUSTAMANTE, Teodoro; JARRÍN, María Cristina. Indicadores sociales y petróleo en la Amazonía. In

BUSTAMANTE, Teodoro (Ed.). La cortina de humo, dinámicas sociales y petróleo en Ecuador. Quito: Flacso, pp. 21-95, 2007.

FONTAINE, Guillaume. ITT: un problema de gobernanza para el Ecuador. In FONTAINE, Guillaume;

FONTAINE, Guillaume. El análisis de políticas en la sociología de la acción pública. Laboratorio de investigación sobre la gobernanza. FLACSO-Ecuador, 2012.

HAUSMANN, Ricardo; HIDALGO, Cesar; BUSTOS, Sebastian; COSCIA, Michele; CHUNG, Sarah; JIMENEZ, Juan; SIMOES, Alexander; YILDIRIM, Muhammed. The Atlas of Economic Complexity. Puritan Press. Cambridge MA, 2011.

JUTEAU-MARTINEAU et al. Ambiente, petróleo y vulnerabilidad política en el oriente ecuatoriano: ¿hacia nuevas formas de gobernanza energética? América Latina Hoy, n. 67, pp. 119-137, 2014.

LLANES, Henry. Estado y Política Petrolera en el Ecuador. Quito: Ecuador, 2004.

LEY ORGÁNICA DE EMPRESAS PÚBLICAS (LOEP). Régimen económico y financiamiento, 2009.

NARVÁEZ, Iván. Represamiento del petróleo en tierra o extracción (eje Ishpingo-Tambococha-Tiputini -ITT - del Yasuní) ¿Un dilema de carácter político? Working paper n. 02, Observatorio Socioambiental FLACSO Ecuador, 2007.

PARRA, Rony. Análisis de la reestructuración institucional del sector petrolero ecuatoriano en el período 2007-2012. Documento de Trabajo, n.02, CEPROEC, 2015.

PAZ Y MIÑO, Juan. Un nuevo ciclo histórico en Ecuador. In La Insignia, 2007.

URL: http://www.lainsignia.org/2007/diciembre/ibe_017.htm. Visitado el 25 noviembre., 2015.

WOOD, Makenzie. Presentación del modelo de gestión de Petroecuador, 2010. 


\section{ANEXOS}

\begin{tabular}{|c|c|c|c|c|}
\hline \multicolumn{5}{|c|}{ TABLA 01: MAYORES PRODUCTORES DE PETRÓLEO Y PRODUCCIÓN PER CÁPITA (2014) } \\
\hline & Países & $\begin{array}{l}\text { Producción de petróleo } \\
\text { (miles de barriles por día) }\end{array}$ & Producción de petróleo per cápita por año & \\
\hline 1 & Estados Unidos & 13973 & 15,82 & $16^{\circ}$ \\
\hline 2 & Arabia Saudita & 11624 & 134,6 & $4^{\circ}$ \\
\hline 3 & Rusia & 10853 & 27,05 & $13^{\circ}$ \\
\hline 4 & China & 4572 & 1,21 & $29^{\circ}$ \\
\hline 5 & Canadá & 4383 & 44,62 & $7^{\circ}$ \\
\hline 6 & EAU & 3471 & 138,35 & $3^{\circ}$ \\
\hline 7 & Irán & 3375 & 15,63 & $17^{\circ}$ \\
\hline 8 & Iraq & 3371 & 34,17 & $9^{\circ}$ \\
\hline 9 & Brasil & 2950 & 5,25 & $24^{\circ}$ \\
\hline 10 & México & 2812 & 8,48 & $19^{\circ}$ \\
\hline 11 & Kuwait & 2780 & 242,54 & $2^{\circ}$ \\
\hline 12 & Venezuela & 2689 & 32,05 & $11^{\circ}$ \\
\hline 13 & Nigeria & 2427 & 4,86 & $26^{\circ}$ \\
\hline 14 & Qatar & 2055 & 310,91 & $1^{\circ}$ \\
\hline 15 & Noruega & 1904 & 133,5 & $5^{\circ}$ \\
\hline 16 & Angola & 1756 & 26,28 & $14^{\circ}$ \\
\hline 17 & Argelia & 1721 & 15,9 & $15^{\circ}$ \\
\hline 18 & Kazakstán & 1719 & 35,63 & $8^{\circ}$ \\
\hline 19 & Colombia & 1016 & 7,66 & $21^{\circ}$ \\
\hline 20 & India & 978 & 0,28 & $30^{\circ}$ \\
\hline 21 & Omán & 951 & 80,87 & $6^{\circ}$ \\
\hline 22 & Indonesia & 911 & 1,3 & $28^{\circ}$ \\
\hline 23 & Reino Unido & 906 & 5,1 & $25^{\circ}$ \\
\hline 24 & Azerbaiyán & 856 & 32,29 & $10^{\circ}$ \\
\hline 25 & Argentina & 715 & 6,05 & $23^{\circ}$ \\
\hline 26 & Malasia & 697 & 8,27 & $20^{\circ}$ \\
\hline 27 & Egipto & 667 & 2,71 & $27^{\circ}$ \\
\hline 28 & Ecuador & 557 & 12,49 & $18^{\circ}$ \\
\hline 29 & Libia & 516 & 30,01 & $12^{\circ}$ \\
\hline 30 & Australia & 478 & 7,28 & $22^{\circ}$ \\
\hline
\end{tabular}

Fuente: Elaboración de los autores con dados de la OPEP, Banco Mundial y US Energy Information Administration (EIA). 


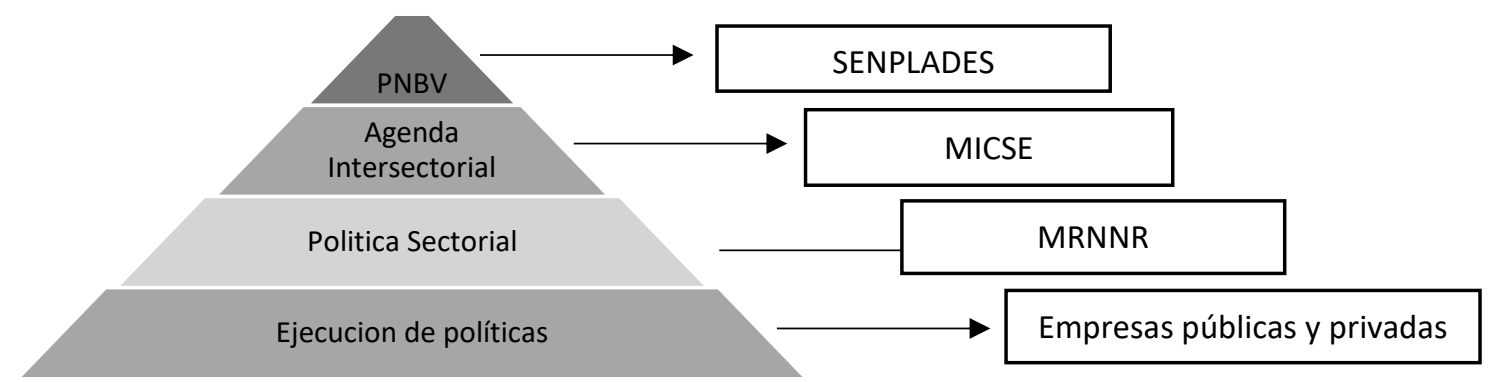

GRÁFICA 01

MODELO INSTITUCIONAL PARA LA CREACIÓN DE POLÍTICAS PÚBLICAS, SECTOR PETROLERO

Fuente: Parra (2015).

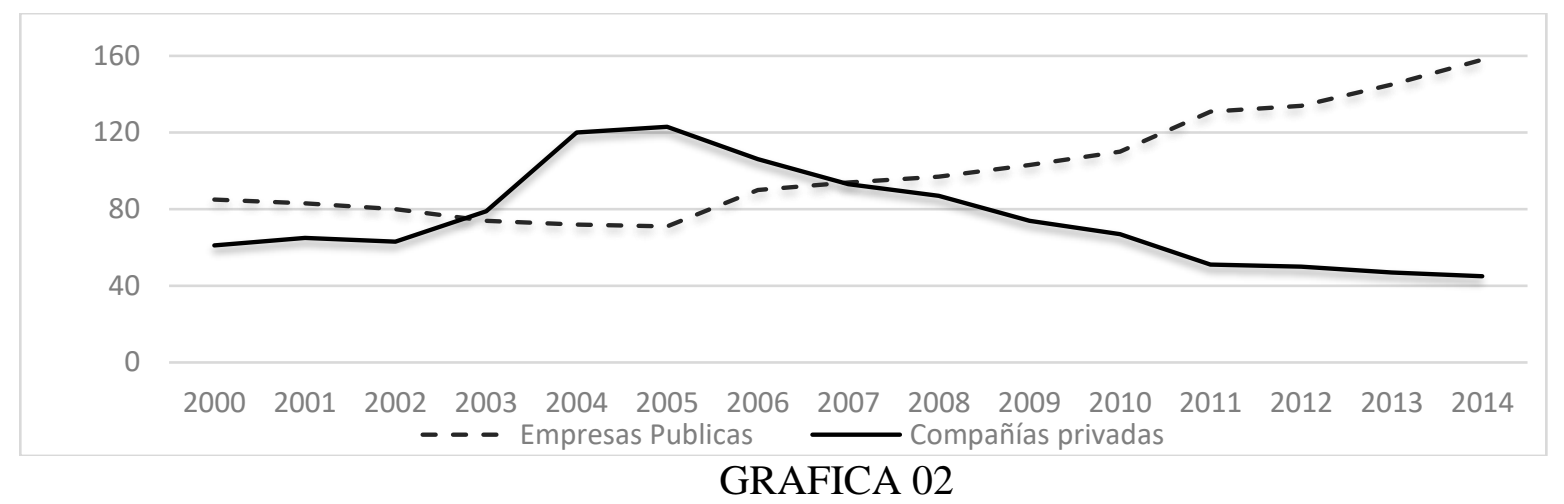

PRODUCCIÓN NACIONAL DE PETRÓLEO EN ECUADOR (MILLONES DE BARRILES)

Fuente: Elaboración de los autores con dados de la EP Petroecuador.

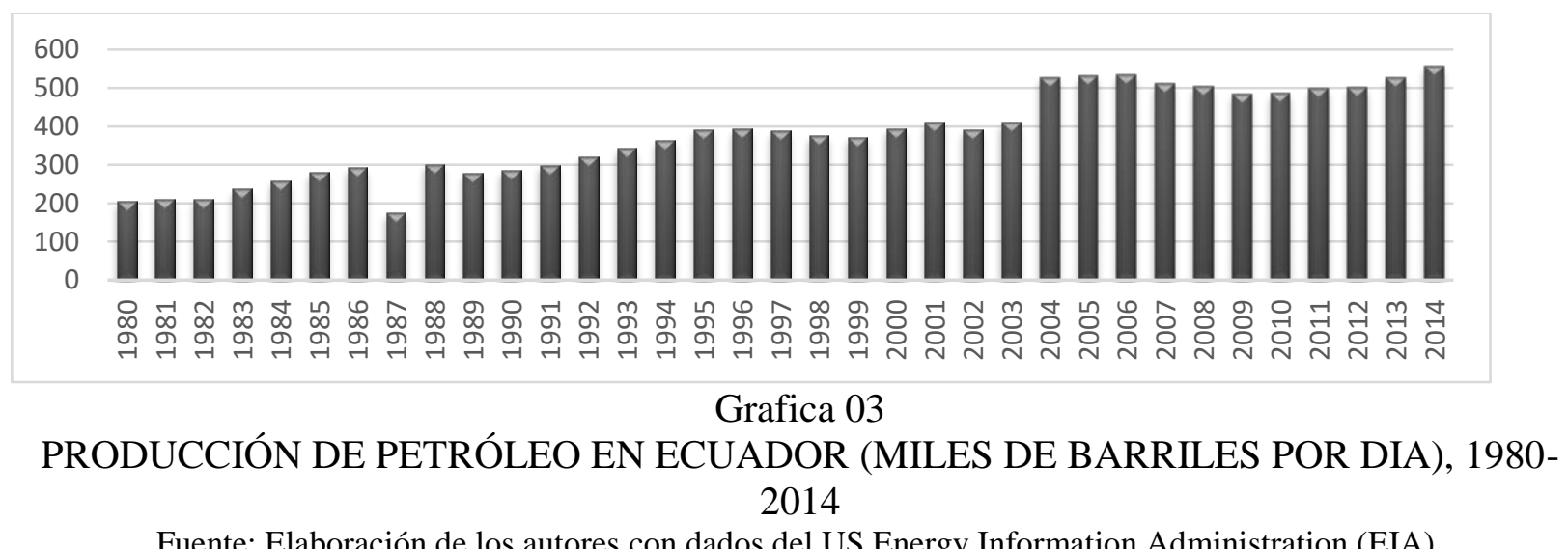

Fuente: Elaboración de los autores con dados del US Energy Information Administration (EIA). 


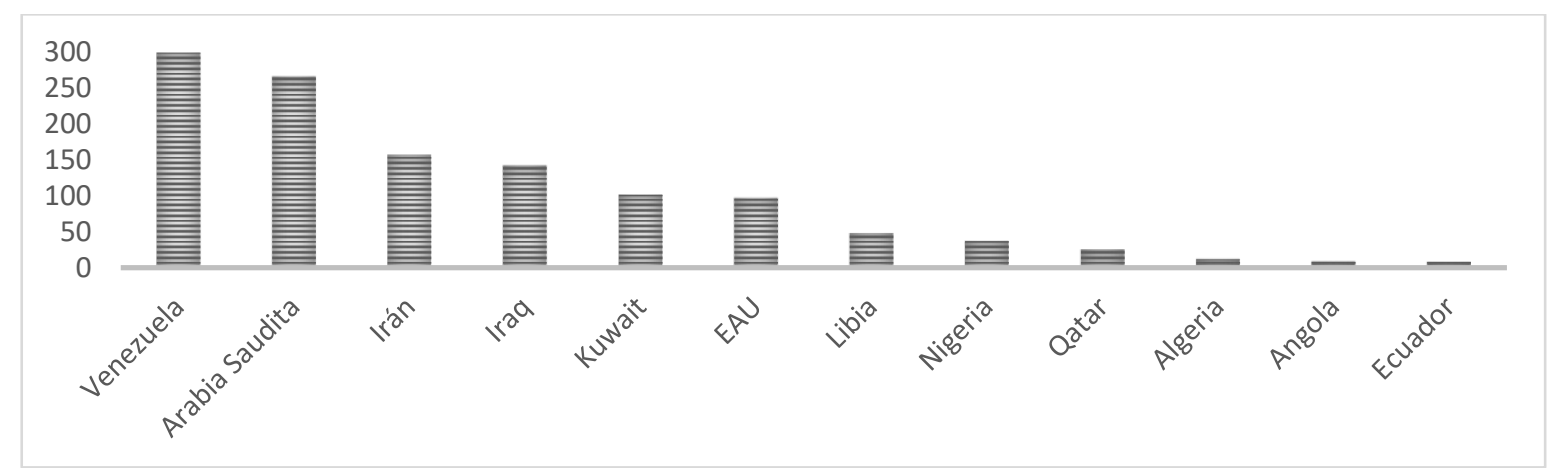

GRAFICA 04

RESERVAS PROBADAS DE PETRÓLEO CRUDO ENTRE LOS MIEMBROS DE LA OPEP (MIL MILLONES DE BARRILES)

Fuente: Elaboración de los autores con dados del boletín estadístico anual de 2015 de la OPEP.

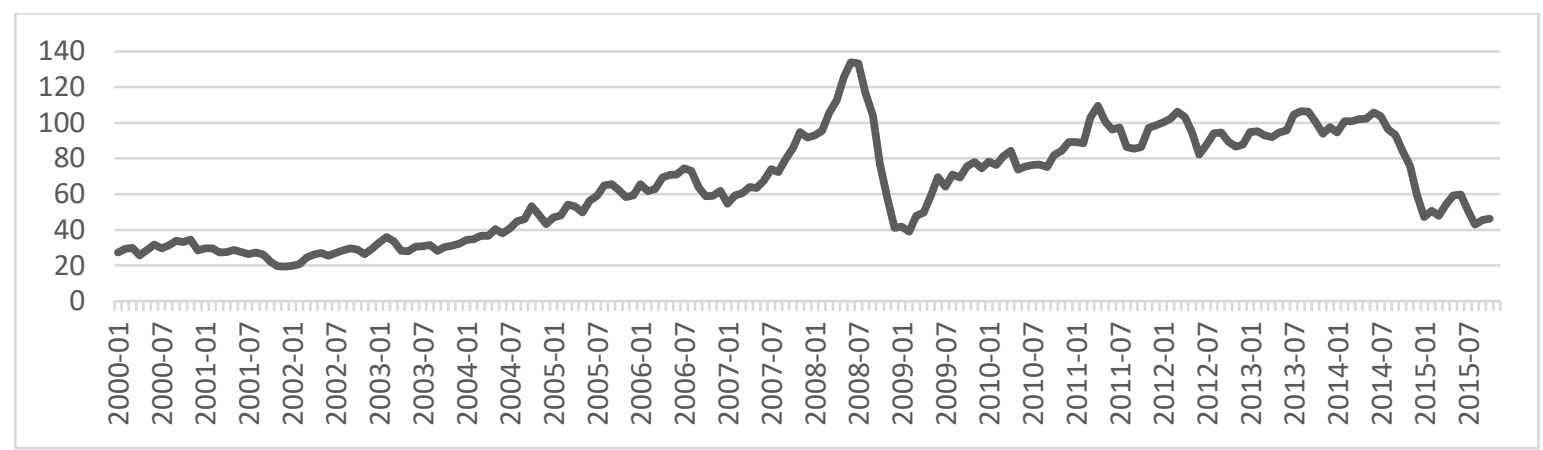

\section{GRAFICA 05}

PRECIO DEL PETRÓLEO (WTI), ENERO 2000-OCTUBRE 2015 (USD)

Fuente: Elaboración de los autores con dados del US Energy Information Administration (EIA). 